\title{
Association between the clinical severity of oral lichen planus and anti-TPO level in thyroid patients
}

\section{Milad ALIKHANI(a) \\ Parichehr GHALAIANI(a) \\ Elham ASKARIYAN ${ }^{(b)}$ \\ Zahra Ahmadi KHUNSARAKI(c) \\ Atefeh TAVANGAR(a) \\ Aliasghar NADERI(a)}

(a) Isfahan University of Medical Sciences, Department of Oral and Maxillofacial Medicine, Isfahan, Iran.

(b)Islamic Azad University, Department of psychology, Najafabad Branch, Najafabad, Iran.

(c)|sfahan University of Medical Sciences, Dental School, Isfahan, Iran.

Declaration of Interests: The authors certify that they have no commercial or associative interest that represents a conflict of interest in connection with the manuscript.

\section{Corresponding Author:}

Aliasghar Naderi

E-mail: aliasgharnederi@ymail.com

DOI: 10.1590/1807-3107BOR-2017.vol31.0010

Submitted: Apr 15, 2016

Accepted for publication: Oct 21, 2016

Last revision: Dec 1, 2016

\begin{abstract}
This study considered a possible relationship between the severity of oral lichen planus (OLP), serum anti-TPO autoantibodies $(\mathrm{TPOAb})$ titer and thyroid disease in OLP patients. Forty-six OLP patients with positive TPOAb results $(>35 \mathrm{IU} / \mathrm{ml}$ ) who had also been diagnosed with thyroid disease were included in the study group. The control group consisted of 46OLP patients with no thyroid disease. The study and control groups (92) were divided to two subgroups of erosive OLP (EOLP) and non-erosive OLP (NEOLP). Serum TPOAb levels and IL-8 (to measure OLP severity) were evaluated using the independent t-test, chi-square and conditional logistic regression analysis $(\alpha=0.05)$. A significant positive correlation was found between serum IL-8 and TPOAb levels in the study group $(r=0.783$; $p=0.001)$. The positive blood levels of TPOAb were significantly associated with an increased risk of EOLP (OR $=4.02$ at 95\%CI; 1.21-13.4; $\mathrm{p}=0.023$ ). It is possible to used positive serum TPOAb levels in patients with OLP as in indicator of possible undetected thyroid disorders in those patients. Because erosive OLP has been associated with TPOAb in thyroid patients, it may be useful to determine TPOAb levels of such patients to diagnose a possible undetected thyroid disorders and follow-up for malignancy.
\end{abstract}

Keywords: Lichen Planus, Oral; Autoimmunity; Thyroid Diseases; Cytokines.

\section{Introduction}

Oral lichen planus (OLP) is a chronic inflammatory disease affecting the oral mucosa that may also involve the skin and the genitalia. ${ }^{1}$ OLP may appear clinically as a reticular, erythematous or erosive type of lesion. ${ }^{2}$ Although the etiopathology of OLP remains unidentified, it is believed that immune dysregulation plays a crucial role. ${ }^{2,3}$ Immune dysregulation in OLP includes unusual production of inflammatory mediators, ${ }^{4}$ of which cytokines are the most prominent. ${ }^{5}$

The abnormal expression pattern of cytokines such as IL-1, -2, -4, -5, -6, -8, $-10,-18$ and TNF- $\alpha$ has been found in the lesions, serum and saliva of patients with OLP. ${ }^{5}$ Elevated levels of IL-8 in the serum of OLP patients have been recorded and reported in several studies. ${ }^{6,7,9,9}$ It has also been reported that the clinical severity of OLP is directly associated with the IL-8 level in the serum. ${ }^{9}$ 
A high frequency of circulating anti-nuclear antibodies (ANA), anti-thyroglobulin antibodies (TGA) and anti-thyroid microsomal autoantibodies (TMA) has been reported in OLP patients. ${ }^{10,11}$ These findings suggest a possible association between autoimmune disease, including thyroid disorders, and OLP. ${ }^{10}$ It is known that autoantibodies against thyroid gland antigenic components such as thyroid peroxidase (TPO) and thyroglobulin (TG) can induce epithelial cell damage ${ }^{12,13}$ and that these autoantibodies are closely linked to Hashimoto's thyroiditis and Graves' disease. ${ }^{14,15}$

Although a causal relationship between OLP and thyroid gland disorders has not been discovered, the association of OLP and thyroid disease has been studied. ${ }^{16}$ It has been suggested that patients with a history of thyroid gland disorders, especially hypothyroidism, are more likely to have OLP than healthy subjects. ${ }^{17,18}$ No studies were found in English-language publications, however, that had found a possible association between the severity of clinical signs of OLP and the serum titer of anti-TPO antibodies (TPOAb) in OLP patients who have also been diagnosed with thyroid disease. The current study examined a possible association between the clinical severity of OLP and the titer of serum TPOAb in OLP patients with thyroid disease.

\section{Methodology}

Because the clinical severity of OLP is associated with elevated serum IL-8 levels, ${ }^{9}$ IL- 8 serves as a reliable tool to assess the clinical severity of this disease. A possible association between the clinical severity of OLP and the titer of serum TPOAb in OLP patients with thyroid disease was determined using serum IL-8 level as an indicator in the present study.

Between November 2010 and December 2013, 340 OLP patients visited the Institute of Dentistry, Isfahan University of Medical Science, Iran. A total of 216 individuals who denied a history of thyroid disease or thyroid medication consumption were excluded by evaluating their self-assessed general health status forms. Also excluded were patients suspected of having drug or dental restoration-related oral lichenoid lesions. An additional 62 OLP patients with areca quid chewing habits, hypertension and autoimmune diseases such as systemic lupus erythematosus, rheumatoid arthritis, Sjögren's syndrome, pemphigus vulgaris and cicatricial pemphigoid were also excluded. In total, 278 (81.5\%) individuals were excluded from the present study.

A total of 62 patients (18.5\%) were included in the initial study group. All participants had a history of taking thyroxin or suffering from thyroid gland disorders according to their self-assessed general health status forms. Diagnosis of OLP in the 62 patients was initially done by clinical impression, which was later confirmed histologically using the most recent diagnostic criteria. ${ }^{19}$

Blood samples were collected from all OLP patients with self-reported thyroid disease $(n=62)$. $\mathrm{TSH}$, thyroxine (T4) and tri-iodothyronine (T3) thyroid function tests were carried and TPOAb and serum IL-8 levels were measured. TPOAb values higher than $35 \mathrm{IU} / \mathrm{ml}$ were considered positive. ${ }^{20}$ Of the 62 patients assessed, $46(74 \%)$ were TPOAb positive and were included in the final study group. The patients were referred to an endocrinologist to confirm the diagnosis of thyroid gland disorder based on the findings of the thyroid gland function tests. Of the 46 patients, 34 were diagnosed with Hashimoto's thyroiditis, 8 with Graves's disease and 4 with hyperthyroidism.

The 46 OLP patients were matched for gender and age with 46 patients having no reported history of thyroid disease to serve as the control group. Because $10 \%$ to $15 \%$ of clinically healthy individuals may have high TPOAb titer, ${ }^{21}$ blood samples were taken from all 46 controls to rule out thyroid malfunction and their TSH, T3, T4, TPOAb and serum IL-8 levels were measured. None recorded positive TPOAb levels $(>35 \mathrm{IU} / \mathrm{ml})$ and all were included in the control group. The Ethics Committee of Isfahan University of Medical Sciences approved the protocol of this study and an informed consent was obtained from each participant.

All OLP patients (46 study and 46 control subjects) were divided into two subgroups based on the clinical nature of their lesions (erosive or non-erosive). 
Non-erosive OLP (NEOLP) was defined based on clinical presentation of radiating grayish-white striae and/or plaque separately or in combination. Any sign of non-traumatic ulceration in the oral mucosa associated with typical lichen signs indicated erosive OLP (EOLP). ${ }^{22}$ The participants underwent evaluations of for TPOAb level using IMMULITE 2000 anti-TPO (EURO/DPC; United Kingdom). IL-8 levels were measured using an Interleukin-8 Elisa kit (Bender MedSystems; Austria).

SPSS (v. 18) was used to analyze the data. The distribution of the variables was examined for normality using the Kolmogorov-Smirnov test. Because the data was normally distributed, a parametric independent $t$-test was carried out. The difference in the presence of TPOAb was compared between the EOLP and NEOLP subjects using a chi-square test. The odds ratios (OR) at a $95 \%$ confidence interval (CI) was calculated using conditional logistic regression adjusted for confounders. The threshold of statistical significance was set at $\mathrm{p}<0.05$.

\section{Results}

Most of the OLP patients $(\mathrm{n}=34 ; 74 \%)$ in each group (study and control) were women. The mean age of the study and control subjects at admission to the university dental clinic was 45 years (17-68) and 45 years (18-72), respectively.

The regular blood levels of TSH were $0.4-4 \mathrm{mIU} / \mathrm{l}$, T4 were $4.5-11.2 \mathrm{mcg} / \mathrm{dl}$ and T3 were $2.3-4.2 \mathrm{pg} / \mathrm{ml}^{20}$ The mean results of the thyroid function tests for each group confirmed that the mean blood levels of the three hormones in the control group were within normal limits (Table 1). The findings were also normal in the

Table 1. Mean level ( \pm SD) of thyroid gland function tests in study and control groups.

\begin{tabular}{lccc}
\hline & \multicolumn{3}{c}{ Thyroid function tests } \\
\cline { 2 - 4 } Group & TSH $(\mathrm{mlU} / \mathrm{l})$ & $\begin{array}{c}\text { Free T4 } \\
(\mathrm{mcg} / \mathrm{dl})\end{array}$ & $\begin{array}{c}\text { Free T3 } \\
(\mathrm{pg} / \mathrm{ml})\end{array}$ \\
\hline Study & $6.87 \pm 0.54$ & $2.63 \pm 0.18$ & $1.9 \pm 0.43$ \\
Control & $1.9 \pm 0.23$ & $7.6 \pm 1.1$ & $3.6 \pm 0.56$ \\
Normal level & $0.5-6$ & $4-11$ & $0.8-18$ \\
\hline
\end{tabular}

individuals of the control group, further verifying that the control group was free of thyroid gland malfunction.

Most ( $n=30,65.4 \%)$ of the 46 OLP patients with positive TPOAb levels (study group) were diagnosed with EOLP; only 16 (34.6\%) were diagnosed with NEOLP. The majority of individuals ( $n=32,69.5 \%)$ in the control group were diagnosed with NEOLP; only $14(30.5 \%)$ were identified with EOLP. The mean TPOAb level in the total study group was $90.3 \pm 18.8(\mathrm{IU} / \mathrm{ml})$; this value was $102 \pm 0.23$ for the NEOLP patients compared to $11 \pm 4.8$ (IU/ml) in the control group. There was a large difference between the study and control groups for serum IL-8 versus TPOAb levels and this difference was statistically significant $(p=0.001)$ (Table 2). A significant positive correlation was also found between serum IL-8 and TPOAb levels in the study group $(r=0.783 ; p=0.001)$. There was no significant correlation between IL-8 and TPOAb levels in the control group $(p=0.65)$.

All OLP patients, regardless of TPOAb status $(\mathrm{n}=92)$ were divided into EOLP $(\mathrm{n}=44)$ and NEOLP $(\mathrm{n}=48)$ groups (Table 3). A statistically significant correlation was found between the occurrence of EOLP and positive TPOAb (> $35 \mathrm{IU} / \mathrm{ml})$ that indicates that TPOAb positivity is significantly associated with an increased chance of EOLP (OR $=4.02$ at $95 \% \mathrm{CI}$; 1.21-13.4; $\mathrm{p}=0.023)$. A significant difference was also found between groups for serum IL-8 level. Increased IL-8 levels were significantly associated with an increased chance of EOLP (OR $=1.276$ at 95\%CI; 1.092-1.49; $\mathrm{p}=0.002$ ).

Table 2. Prevalence of clinical presentations of oral lichen planus (OLP) and the mean anti-TPO autoantibodies (TPOAb) serum levels $(\mathrm{IU} / \mathrm{ml}$ ) and IL-8 $(\mathrm{pg} / \mathrm{ml})$ in study and control groups.

\begin{tabular}{cccccc}
\hline \multicolumn{5}{c}{ Type of OLP } & \multicolumn{2}{c}{ Serum markers } & Marker \\
Group & $\begin{array}{c}\text { EOLP } \\
(\%)\end{array}$ & $\begin{array}{c}\text { NEOLP } \\
(\%)\end{array}$ & TPOAb & IL-8 & correlation \\
\cline { 2 - 5 } Study & 30 & 16 & $90.3 \pm 18.8$ & $12.39 \pm 2.65$ & $\begin{array}{c}r=0.783 ; \\
p=0.001\end{array}$ \\
& $(65.4)$ & $(34.6)$ & & & \\
Control & 14 & 32 & $11 \pm 4.8$ & $3.9 \pm 1.7$ & $\mathrm{p}=0.65$ \\
\hline
\end{tabular}

EOLP: erosive OLP; NEOLP: non-erosive OLP. 
Table 3. Mean levels of serum positive* anti-TPO autoantibodies (TPOAb) and IL-8 (pg/ml) serum levels in patients with EOLP and NEOLP

\begin{tabular}{|c|c|c|c|c|}
\hline \multirow{3}{*}{$\operatorname{OLP}(n=92)^{*}$} & TPOAb & Pos. TPOAb/EOLP & \multirow{3}{*}{$\begin{array}{c}\text { Serum IL-8 level (pg/ml) } \\
\pm \text { SD }\end{array}$} & IL-8 levels/EOLP \\
\hline & pos./neg. ${ }^{* *}$ & \multirow{2}{*}{ OR (\% Cl) } & & \multirow{2}{*}{ OR $(95 \% \mathrm{Cl})$} \\
\hline & $(\%)$ & & & \\
\hline \multirow{2}{*}{$\operatorname{EOLP}(n=44)$} & $30 / 14$ & 4.02 & \multirow{2}{*}{$10.45 \pm 4.1^{*}$} & \multirow{4}{*}{$\begin{array}{c}1.276 \\
{[1.092-1.49]} \\
(p=0.002 \backslash 0\end{array}$} \\
\hline & $(68 / 31)$ & {$[1.21-13.4]$} & & \\
\hline \multirow{2}{*}{$\operatorname{NEOLP}(n=48)$} & $16 / 32$ & $(p=0.023)$ & \multirow{2}{*}{$6.04 \pm 3.93 *$} & \\
\hline & $(33 / 66)$ & & & \\
\hline
\end{tabular}

*Study and control groups; **Values higher than $35 \mathrm{IU} / \mathrm{ml}$ (all 46 were in study group); OLP: oral lichen planus; EOLP: erosive OLP; NEOLP: non-erosive OLP.

\section{Discussion}

The current study examined the association between the clinical severity of OLP and TPOAb titer in patients with thyroid disease. The results indicate that the severity of the clinical expression of OLP lesions were directly linked to the level of serum IL-8, as previously reported, ${ }^{9}$ and also to the level of TPOAb.

Cytokines play an important role in controlling the direction, extent and duration of immune response. ${ }^{23}$ Abnormal expression of cytokines may lead to the onset of autoimmunity. ${ }^{23,24}$ IL-8 plays affects pathogenesis of inflammation ${ }^{25}$ and has the capacity to recruit $T$ cells as well as non-specific inflammatory cells to the sites of inflammation by activating neutrophils. ${ }^{26}$ Serum IL-8 levels are sensitive markers that can be used to monitor disease activity and the severity of OLP. ${ }^{9}$ The level of serum IL-8 also appears to signify an association with reticular and erosive forms of OLP. ${ }^{9}$ Because IL-8 is a pluripotent pro-tumorigenic cytokine that is known to induce angiogenesis, tumor cell proliferation and tumor cell migration, ${ }^{27,28,29}$ evaluation of IL-8 serum level may also serve as an indicator of the severity of OLP and the risk of the developing malignancies.

The results of the current study confirm the initial hypothesis that there is a significant association between the clinical severity of OLP and the level of TPOAb in patients with thyroid disease; however, logical bias may exist in the extent of the differences because the initial inclusion criteria stipulated that TPOAb levels should be higher in the study group than in the control group. The present study showed that IL-8 serum levels were significantly higher in OLP patients with high TPOAb levels who had also been diagnosed with thyroid disease compared to OLP patients with no thyroid disease. The current findings suggest that OLP patients who have positive levels of TPOAb are about four times more likely to develop EOLP than patients with negative TPOAb levels, although this assumption requires further study combined with long-term patient follow-ups. The finding of a correlation between EOLP and TPOAb further supports the conclusion of Chang et al. ${ }^{10}$ who reported that patients with severe clinical EOLP have a significant probability of development of autoimmune markers for thyroid disease such as abnormal TGA and TMA.

There was a substantial difference between the study and control groups for serum IL-8 level. Karanikas et al. ${ }^{30}$ showed that TPOAb titer correlates with increased presence of $\mathrm{T}$ cells that produce cytokines, which are responsible for cell damage. They also found increased production of TNF-a by CD8+ cytotoxic T lymphocytes in patients with high TPOAb titer. ${ }^{30}$ Because, in many cell types, synthesis of IL-8 is strongly stimulated by TNF-a, ${ }^{31}$ it appears reasonable for IL-8 levels to be significantly higher in OLP patients with positive TPOAb values and thyroid disease. This may also explain the increased prevalence of EOLP in patients with thyroid disease than in OLP patients free of thyroid disease. The histological findings show that there was very high neutrophilic infiltration of the epithelium in EOLP than in other forms of the disease. ${ }^{32}$ This infiltration 
can, at least partially, be attributed to the higher level of IL-8 in EOLP patients.

The present study showed that 46 of the 340 initial patients $(14 \%)$ had thyroid disease with positive $\mathrm{TPOAb}$ values. Similar findings were reported by Lo Muzio et al. ${ }^{16}$ and Siponen et al., ${ }^{18}$ who found a history of thyroid gland pathosis in $14 \%$ and $15 \%$ of OLP cases, respectively. Although a causal relationship between OLP and thyroid disease was not investigated in the current study, it can be speculated that circulating thyroid antibodies may trigger an autoimmune response in the oral mucosa and cause development of OLP. This immune response could

\section{References}

1. Eisen D, Carrozzo M, Bagan Sebastian JV, Thongprasom K. Oral lichen planus: clinical features and management. Oral Dis. 2005;11(6):338-49. doi: 10.1111/j.1601-0825.2005.01142.x

2. Lodi G, Scully C, Carrozzo M, Griffiths M, Sugerman $\mathrm{PB}$, Thong prasom K. Current controversies in oral lichen planus: report of an international consensus meeting. Part 1. Viral infections and etiopathogenesis. Oral Surg Oral Med Oral Pathol Oral Radiol Endod. 2005;100(1):40-51. doi:10.1016/j.tripleo.2004.06.077

3. Sugerman PB, Savage NW, Walsh LJ, Zhao ZZ, Zhou XJ, Khan A et al. The pathogenesis of oral lichen planus. Crit Rev Oral Biol Med. 2002;13(4):350-65. doi:10.1177/154411130201300405

4. Tao XA, Li CY, Rhodus NL, Xia J, Yang XP, Cheng B. Simultaneous detection of IFN-gamma and IL-4 in lesional tissues and whole unstimulated saliva from patients with oral lichen planus. J Oral Pathol Med. 2008;37(2):83-7. doi:10.1111/j.1600-0714.2007.00593.x

5. Lu R, Zhang J, Sun W, Du G, Zhou G. Inflammation-related cytokines in oral lichen planus: an overview. J Oral Pathol Med. 2015;44(1):1-14. doi:10.1111/jop.12142

6. Rhodus NL, Cheng B, Bowles W, Myers S, Miller L, Ondrey F. Proinflammatory cytokine levels in saliva before and after treatment of (erosive) oral lichen planus with dexamethasone. Oral Dis. 2006;12(2):112-6. doi:10.1111/j.1601-0825.2005.01165.x

7. Rhodus NL, Cheng B, Myers S, Miller L, Ho V, Ondrey F. The feasibility of monitoring NF-kappaB associated cytokines: TNF-alpha, IL-1alpha, IL-6, and IL-8 in whole saliva for the malignant transformation of oral lichen planus. Mol Carcinog. 2005;44(2):77-82. doi:10.1002/mc.20113 also occur in cases in which OLP precedes the onset of thyroid dysfunction; ${ }^{16}$ therefore further studies on the association between thyroid disease and OLP are required.

In conclusion, because the erosive pattern of OLP is associated with positive findings of TPOAb in patients with thyroid disease, it could be a clinically useful method of determining TPOAb levels in patients with EOLP to allow diagnosis of hidden thyroid disorders. The high levels of TPOAb found may indicate high levels of IL-8 and point to increased risk of development of malignancies and warrants much closer follow-ups.

8. Zhang Y, Lin M, Zhang S, Wang Z, Jiang L, Shen J et al. NF-kappaB-dependent cytokines in saliva and serum from patients with oral lichen planus: a study in an ethnic Chinese population. Cytokine. 2008;41(2):144-9. doi:10.1016/j.cyto.2007.11.004

9. Sun A, Wang JT, Chia JS, Chiang CP. Serum interleukin-8 level is a more sensitive marker than serum interleukin-6 level in monitoring the disease activity of oral lichen planus. Br J Dermatol. 2005;152(6):1187-92. doi:10.1111/j.1365-2133.2005.06497.x

10. Chang JY, Chiang CP, Hsiao CK, Sun A. Significantly higher frequencies of presence of serum autoantibodies in Chinese patients with oral lichen planus. J Oral Pathol Med. 2009;38(1):48-54. doi:10.1111/j.1600-0714.2008.00686.x

11. Lin HP, Wang YP, Chia JS, Sun A. Modulation of serum anti-thyroglobulin and anti-thyroid microsomal autoantibody levels by levamisole in patients with oral lichen planus. J Formos Med Assoc. 2011;110(3):169-74. doi:10.1016/S0929-6646(11)60027-2

12. Rodien P, Madec AM, Ruf J, Rajas F, Bornet H, Carayon P et al. Antibody-dependent cell-mediated cytotoxicity in autoimmune thyroid disease: relationship to antithyroperoxidase antibodies. J Clin Endocrinol Metab. 1996;81(7):2595-600. doi:10.1210/jcem.81.7.8675583

13. Chiovato L, Bassi P, Santini F, Mammoli C, Lapi P, Carayon $P$ et al. Antibodies producing complement-mediated thyroid cytotoxicity in patients with atrophic or goitrous autoimmune thyroiditis. J Clin Endocrinol Metab. 1993;77(6):1700-5. doi:10.1210/jcem.77.6.7903315

14. Bjoro T, Holmen J, Krüger O, Midthjell K, Hunstad K, Schreiner $\mathrm{T}$ et al. Prevalence of thyroid disease, thyroid dysfunction and thyroid peroxidase antibodies in a large, unselected population. Eur J Endocrinol. 2000;143(5):639-47. doi:10.1530/eje.0.1430639 
15. Prummel MF, Wiersinga WM. Thyroid peroxidase autoantibodies in euthyroid subjects. Best Pract

Res Clin Endocrinol Metab. 2005;19(1):1-15. doi:10.1016/j.beem.2004.11.003

16. Lo Muzio L, Santarelli A, Campisi G, Lacaita M, Favia G. Possible link between Hashimoto's thyroiditis and oral lichen planus: a novel association found. Clin Oral Investig. 2013;17(1):333-6. doi:10.1007/s00784-012-0767-4

17. Garcia-Pola MJ, Llorente-Pendás S, Seoane-Romero JM, Berasaluce MJ, García-Martín JM. Thyroid disease and oral lichen planus as comorbidity: a prospective case-control study. Dermatology. 2016;232(2):214-9. doi:10.1159/000442438

18. Siponen M, Huuskonen L, Läärä E, Salo T. Association of oral lichen planus with thyroid disease in a Finnish population: a retrospective case-control study. Oral Surg Oral Med Oral Pathol Oral Radiol Endod. 2010;110(3):319-24. Doi:10.1016/j.tripleo.2010.04.001

19. Meij EH, Waal I. Lack of clinicopathologic correlation in the diagnosis of oral lichen planus based on the presently available diagnostic criteria and suggestions for modifications. J Oral Pathol Med. 2003;32(9):507-12. Doi:10.1034/j.1600-0714.2003.00125.x

20. Gardner D. Normal hormone reference ranges. In: Gardner DG, Shoback D. Greenspan's basic \& clinical endocrinology. $9^{\text {th }}$ ed. McGraw-Hill; 2011. Appendix.

21. Chardès T, Chapal N, Bresson D, Bès C, Giudicelli V, Lefranc MP et al. The human anti-thyroid peroxidase autoantibody repertoire in Graves' and Hashimoto's autoimmune thyroid diseases. Immunogenetics. 2002;54(3):141-57. Doi:10.1007/s00251-002-0453-9

22. DeRossi SS1, Ciarrocca KN. Lichen planus, lichenoid drug reactions, and lichenoid mucositis. Dent Clin North Am. 2005;49(1):77-89. Doi:10.1016/j.cden.2004.08.004

23. Roescher N, Tak PP, Illei GG. Cytokines in Sjogren's syndrome. Oral Dis. 2009;15(8):519-26. Doi:10.1111/j.1601-0825.2009.01582.x

24. Moudgil KD, Choubey D. Cytokines in autoimmunity: role in induction, regulation, and treatment. J Interferon Cytokine Res. 2011;31(10):695-703. Doi:10.1089/jir.2011.0065
25. Dobreva I, Waeber G, James RW, Widmann C. Interleukin- 8 secretion by fibroblasts induced by low density lipoproteins is p38 MAPK-dependent and leads to cell spreading and wound closure. J Biol Chem. 2006;281(1):199-205. Doi:10.1074/jbc.M508857200

26. Schröder JM. The neutrophil-activating peptide $1 /$ interleukin 8, a novel neutrophil chemotactic cytokine. Arch Immunol Ther Exp (Warsz). 1992;40(1):23-31.

27. Silverman S Jr, Gorsky M, Lozada-Nur F. A prospective follow-up study of 570 patients with oral lichen planus: persistence, remission and malignant association. Oral Surg Oral Med Oral Pathol. 1985;60(1):30-4 Doi:10.1016/0030-4220(85)90210-5

28. Arihiro K, Oda H, Kaneko M, Inai K: Cytokines facilitate chemotactic motility of breast carcinoma cells. Breast Cancer. 2000;7(3):221-30. Doi:10.1007/BF02967464

29. Singh RK, Gutman M, Radinsky R, Bucana CD, Fidler IJ. Expression of interleukin 8 correlates with the metastatic potential of human melanoma cells in nude mice. Cancer Res. 1994;54(12):3242-7

30. Karanikas G, Schuetz M, Wahl K, Paul M, Kontur S, Pietschmann P et al. Relation of anti-TPO autoantibody titre and T-lymphocyte cytokine production patterns in Hashimoto's thyroiditis. Clin Endocrinol (Oxf). 2005;63(2):191-6. doi:10.1111/j.1365-2265.2005.02324.x

31. Osawa Y, Nagaki M, Banno Y, Brenner DA, Asano T, Nozawa $Y$ et al. Tumor necrosis factor alpha-induced interleukin-8 production via NF-kappaB and phosphatidylinositol 3-kinase/Akt pathways inhibits cell apoptosis in human hepatocytes. Infect Immun. 2002;70(11):6294-301. doi:10.1128/IAI.70.11.6294-6301.2002

32. Fernández-González F, Vázquez-Álvarez R, Reboiras-López D, Gándara-Vila P, García-García A, Gándara-Rey JM. Histopathological findings in oral lichen planus and their correlation with the clinical manifestations. Med Oral Patol Oral Cir Bucal. 2011;16(5):641-6. doi:10.4317/medoral.16983 